\title{
A case of delayed diagnosis of Necrolytic Migratory Erythema
}

wissal abdelli ${ }^{1}$, Fatima Alaoui ${ }^{1}$, Asmahene Souissi ${ }^{1}$, Wiem Sassi ${ }^{1}$, Ines Chelly ${ }^{1}$, Slim Haouet $^{1}$, and Mourad Mokni ${ }^{1}$

${ }^{1}$ Rabta Hospital

August 4, 2021

\begin{abstract}
Necrolytic Migratory Erythema (NME) is a rare cutaneous paraneoplastic manifestation of glucagonoma. We report a case of a woman with a 6-year history of delayed diagnosis of Glucagonoma. This case highlights the atypical clinical features of NME which makes the diagnosis difficult.
\end{abstract}

Title: A case of delayed diagnosis of Necrolytic Migratory Erythema

Wissal ABDELLI ${ }^{1}$, Fatima ALAOUI ${ }^{1}$, Asmahen SOUISSI ${ }^{1}$, Wiem SASSI $^{1}$, Ines $_{\text {CHELLY }}{ }^{2}$, Slim HAOUET $^{2}$, Mourad MOKNI ${ }^{1}$

1-Dermatology Department - Rabta hospital, Tunis, Tunisia

2-Anatomopathology Department - Rabta hospital, Tunis, Tunisia

Corresponding author's address:

Wissal ABDELLI, MD

Address: Rabta hospital Dermatology Department, Jebal Lakhdhar street 1007- La Rabta Jebbari -Tunis -Tunisia.

Tel/ Fax: 0021671572155

E-mail:abdelliwissal7@gmail.com

Key words : glucagonoma, necrolytic migratory erythema, paraneoplastic, glucagonoma syndrome

Conflict of interest : The authors have no conflict of interest to declare

Statement: This article has no funding source

Number of words: 1023

Number of references: 17

Number of figures: 3

\section{Key clinical message}

Our case highlights the atypical clinical features of Necrolytic migratory erythema which makes the diagnosis difficult. Dermatologists, must be aware of this condition subsequently they can be the first physician to suspect it.

Abstract 
Necrolytic Migratory Erythema (NME) is a rare cutaneous paraneoplastic manifestation of glucagonoma. We report a case of a woman with a 6-year history of delayed diagnosis of Glucagonoma. This case highlights the atypical clinical features of NME which makes the diagnosis difficult.

\section{Case report}

\section{Introduction}

Necrolytic migratory erythema (NME) is a rare cutaneous paraneoplastic manifestation of glucagonoma. Estimated incidence of glucagonoma is $1 / 20,000,000 /$ year(1). Elevated glucagon level can cause a variety of clinical manifestations including weight loss, diabetes, and NME. This skin rash has a cyclic nature and lesions exist simultaneously at different stages(2). Misdiagnosis of paraneoplastic cutaneous manifestation could delay the diagnosis of glucagonoma. We report an interesting case of a female patient with a 6-year history of delayed diagnosis of Glucagonoma.

\section{Case report}

A 36-year-old Tunisian woman had been followed in our dermatology department for six years. She was referred to us for chronic prurigo. Laboratory testing revealed hypochromic microcytic anemia. Serum glucose, HBA1C and liver plasma tests were normal. Abdominal ultrasound was normal. She was treated with topical steroid intermittently with variable response. Since this episode, she has returned several times with polymorphous skin lesions such as papules, erythematosquamous and crusty plaques, vesicles, pustules and erosions accompanied by severe pruritus. At that time, we thought she had prurigo (Fig.1a), eczema (Fig.1b), insect bites (Fig.1c) or even drug eruption (Fig.1d).

The eruption was episodic with spontaneous exacerbations and remissions. The patient had no abdominal pain, gastrointestinal symptoms nor weight loss. Multiple skin biopsies were performed. They concluded to drug eruption, eczema, prurigo and erythema multiforme. During the course of the outbreaks, the patient developed angular cheilitis and gingivitis, a deep vein thrombosis in the leg, and psychological problems which affected her social and professional life. Six years later, she presented with acute abdominal and pelvic pain. Abdominal Computed Tomography (CT) was performed and hyperdense mass was confirmed on body-tail pancreatic of $15 \mathrm{~cm}$ in maximum diameter with mild degree of contrast enhancement. Removal of the tumour was indicated and the cutaneous lesions vanished one week after surgery. Pathology report indicated a tumor in the pancreatic alpha cells. Immunohistochemistry showed expression of glucagon and chromogranin A in tumor cells (diagnosis of glucagonoma). No metastases were detected.

Retrospectively, on reviewing the patient's photos, in addition to the deceptive lesions, our patient presented a unique clinically and histologically typical episode in which we missed unfortunately the diagnosis. She had an annular-circinate, erythematous, scaly rash with areas of hyperpigmentation and skin sloughing, mainly involving the extremities, buttocks, and perineum (Fig.2a). The lesions were highly suggestive of NME. Skin biopsy revealed psoriasiform acanthosis and abrupt necrosis of the upper layers of stratum; whereas the lower half of epidermis appears viable, the detached necrolytic portion appears pale with pyknotic nuclei. Perivascular lymphocytic infiltration and scattered extravasated red blood cells were present in the upper dermis (Fig.2b).

The absence of diabetes and gastrointestinal symptoms led to misdiagnosis.

Two years after surgery, the patient presented with typical skin signs of NME (Fig.3) and diabetes. Magnetic resonance imaging (MRI) and abdominal CT were normal. We asked for a review of the MRI and CT scan because we were sure that the tumour had recurred. Two nodules were visualized on MRI: a retropancreatic nodule $(13 \mathrm{~mm})$ and a nodule opposite the tail of the pancreas $(11 \mathrm{~mm})$, with heterogeneous T2 signal, diffusum hypersignal, without intense arterial enhancement, homogeneous at both portal and late phases. The octreoscan didn't show distant metastases. The patient was referred to surgery for surgical resection of the tumour.

\section{Discussion}


Glucagonoma syndrome (GS) is a rare paraneoplastic phenomenon. Its most common features are weight loss, NME and diabetes mellitus (3).NME is considered a hallmark clinical sign of glucagonoma syndrome, present in approximately $70 \%$ of patients (3). The dermatosis evolves in 7-14 days, occurring in spontaneous exacerbations and remissions(4). Cutaneous features can mimic bullous dermatitis; it may also present as psoriasiform or eczematous plaques(5). Our patient's cutaneous lesion were under recognized and we misinterpreted the skin manifestations as more common entities such as prurigo, eczema, and drug eruption for six years. The polymorphism of the cutaneous features and the rarety of glucagonoma delayed the recognition of the clinical syndrome. There are many reports of delayed diagnosis of glucagonoma due to misdiagnosis or delayed diagnosis of skin lesions(6-11). The average time from recognized symptoms to diagnosis is about four years (12). Histology may show non specific dermatitis, requiring multiple biopsies to confirm the diagnosis. The most specific feature includes superficial epithelial necrosis of the upper spinous layer with vacuolated keratinocytes. Lack of specific findings on biopsy and the rarity of the pancreatic tumor can attribute to delays in diagnosis(4).

NME is a valuable cutaneous presentation in this diseases, when the skin lesions relapsed, we were convinced of the recurrence of the tumour even though the scan was normal.

By the time of diagnosis, $50-100 \%$ of patients already present with metastatic disease, and a cure is often impossible(2). However, since this islet cell tumor is slow-growing, prolonged survival (more than 20 years) is possible, and in metastatic disease, most causes of death appear to be unrelated to the tumor(13). Causes of death are correlated to thromboembolism, sepsis, and gastrointestinal bleeding (14).Skin changes appear early in the course of GS and are reason for seeking medical help for the first time. They are followed by systemic symptoms such as weight loss, diarrhea, diabetes mellitus, neuropsychiatric disorders, anemia, and thrombosis.

Diabetes mellitus is found in $80 \%$ of patients with the GS (3). Our patient had no diabetes and this delayed the recognition of the clinical syndrome.

Glucagonoma syndrome is associated with a high incidence of thromboembolism, estimated between 10 and $30 \%$ of patients (15) which is responsible for the immediate cause of death in up to $50 \%$ of patients (16). Our patient was diagnosed with deep vein thrombosis in the leg.

Complete resection of the tumor is the best treatment. Our patient's cutaneous lesions vanished one week after surgery. Patients who underwent resection had longer median survival than patients who did not receive surgery, even when diagnosed with later stages of disease(17).

\section{Conclusion}

The observed patient was diagnosed with a high delayed diagnosis but fortunately before metastases occurred. It highlights the atypical clinical features and non-specific histology of NME which makes the diagnosis difficult. The absence of diabetes, the polymorphism of clinical and histologic presentation, the psychological features contribute to erroneous and delayed diagnosis in our patient.

\section{Author Contributions}

1. ABDELLI Wissal : Writing the article

2. SOUISSI Asmahen, ALAOUI Fatima, SASSI Wiem: Been involved in drafting the manuscript and revising it critically

3. CHELLY Ines, HAOUET Slim : Have made contributions to examine the histology slides

4. Mokni Mourad : Given final approval of the version to be published

\section{References}

1. Jishu Wei, Shibo Lin, Cong Wang, Junli Wu, Zhuyin Qian, Cuncai Dai, et al. Glucagonoma syndrome: A case report. Oncol Lett. 2015;10 (2):1113-6 
2. Teixeira RC, Nico MMS, Ghideti AC. Necrolytic migratory erythema associated with glucagonoma: a report of 2 cases. Clinics. 2008;63(2):267-70.

3. Beek A PV, Haas ERMD, Vloten WAV, Lips CJM, Roijers JFM, Dijk MRC. The glucagonoma syndrome and necrolytic migratory erythema: a clinical review. Eur J Endocrinol. 2004;151:531-7.

4. Tolliver S, Graham J, Kaffenberger BH. A review of cutaneous manifestations within glucagonoma syndrome: necrolytic migratory erythema. Int J Dermatol. 2018;57(6):642-5.

5. Khoshnia M, Norouzi A, Hosseininejad S-M, Hosseininejad F-S. Glucagonoma Neuroendocrine Tumor with the Clue of Necrolytic Migratory Erythematous Rash: A Case Report. 2018;2(1):2292-5.

6. Wu S, Bai J, Xu J, Ma Q, Wu Z. Necrolytic migratory erythema as the first manifestation of pancreatic neuroendocrine tumor. World J Surg Oncol. 2014;12(1):220.

7. Halvorson SAC, Gilbert E, Hopkins RS, Liu H, Lopez C, Chu M, et al. Putting the Pieces Together: Necrolytic Migratory Erythema and the Glucagonoma Syndrome. J Gen Intern Med. 2013;28(11):1525-9.

8. Afsharfard A, Atqiaee K, Lotfollahzadeh S, Alborzi M, Derakhshanfar A. Necrolytic Migratory Erythema as the First Manifestation of Glucagonoma. Case Rep Surg.2012.

9. Han X, Wang D, Kuang T, Rong Y, Lou W. Glucagonoma syndrome: report of one case. Transl Gastro enterol Hepatol. 2016;1.

10. Cruz-Bautista I, Lerman I, Perez-Enriquez B, Padilla LS, Torres CL, Lopez A, et al. Diagnostic challenge of glucagonoma: case report and literature review. Endocr Pract. 2006;12(4):422-6.

11. Sheng Fang, Shuang Li, Tao Cai. Glucagonoma syndrome: a case report with focus on skin disorders. Onco Targets Ther. 2014;7:1449-53.

12. Wermers RA, Fatourechi V, Wynne AG, Kvols LK, Lloyd RV. The Glucagonoma Syndrome Clinical and Pathologic Features in 21 Patients: Medicine (Baltimore).1996;75(2):53-63.

13. el Darouty M, Abu el Ela M. Necrolytic migratory erythema without glucagonoma in patients with liver disease. J Am Acad Dermatol. 1996;34(6):1092-3.

14. Dinc B, Sahin C. Metastatic glucagonoma. Eurasian J Med. 2009;41(1):70.

15. Feldmann R, Wahl S, Steiner A. Normoglycemic glucagonoma syndrome associated with necrolytic migratory erythema. J Eur Acad Dermatol Venereol JEADV. 2018;32(8):e306-7.

16. Tremblay C, Marcil I. Necrolytic Migratory Erythema: A Forgotten Paraneoplastic Condition. J Cutan Med Surg. 2017;21(6):559-61.

17. Cardoso D, Cardoso A, Filho FC. Pancreatic glucagonoma associated with necrolytic migratory erythema: case report and clinical review. Eur J Cancer. 2017;72:S151.

Figures legend

Fig.1 : atypical episodes mimicking other diagnoses

Fig.1a : prurigo-like eruption

Fig.1b : Eczema-like eruption

Fig.1c : Insect bites-like eruption

Fig.1d : drug eruption-like episode

Fig.2a : Necrolytic migratory erythema with typical scaling and erythema.

Fig.2b : Skin biopsy in necrolytic migratory erythema showing a zone of necrolysis in the upper epidermis 
Fig.3 : Erythematous polycyclic lesions with scaling margins
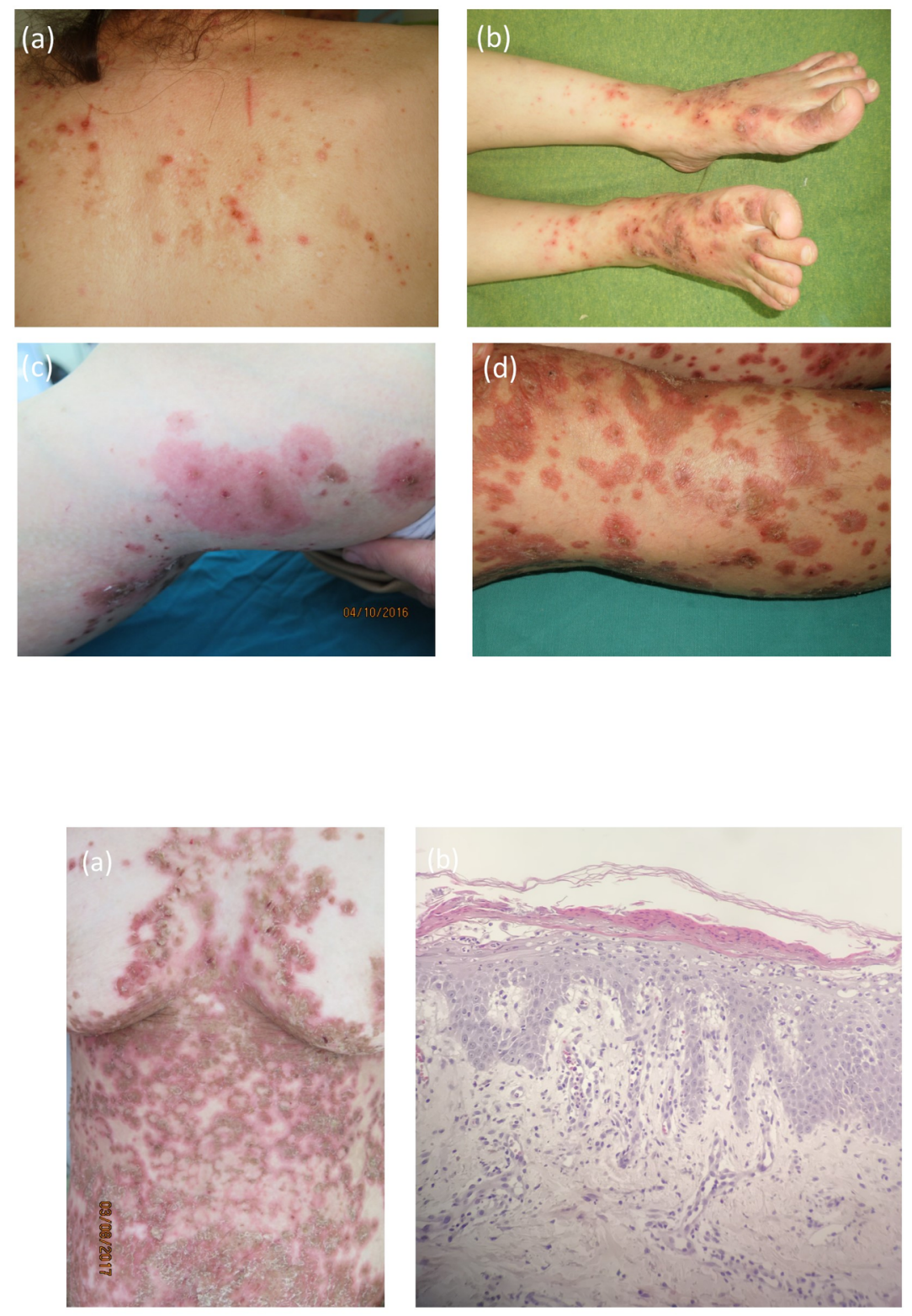


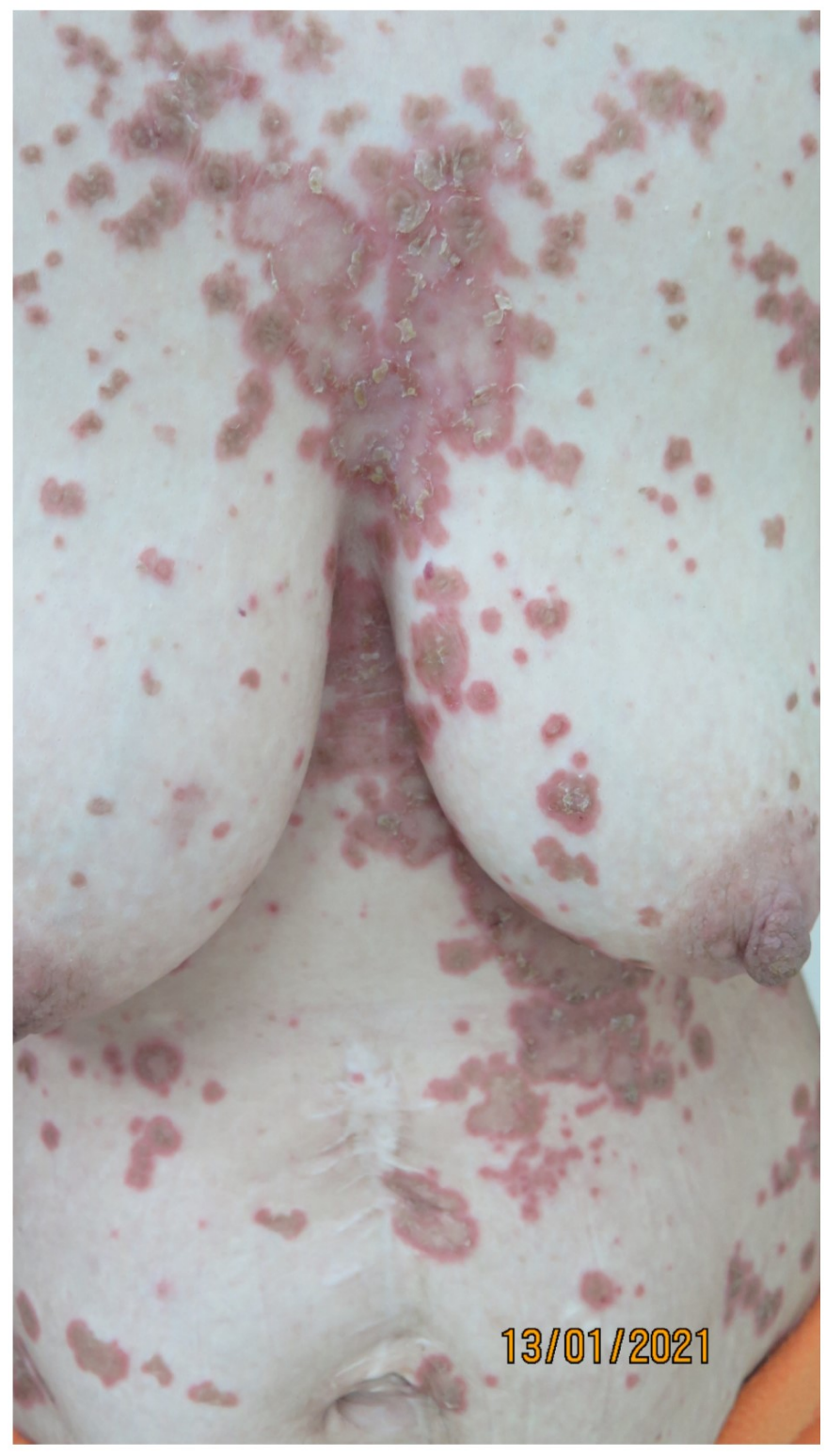

